\title{
Petroleum reservoir simulation using EbFVM: the negative transmissibility issue
}

\author{
C. R. Maliska, J. Cordazzo \& A. F. C. Silva \\ Computational Fluid Dynamics Laboratory, \\ Mechanical Engineering Department, \\ Federal University of Santa Catarina, Florianópolis, SC, Brazil
}

\begin{abstract}
Pioneer methods for simulating petroleum reservoirs were developed in the framework of finite difference methods with Cartesian grids. Therefore, the concept of transmissibility was readily applied for calculating the fluxes at the control volume interfaces. With the advent of new methods using curvilinear non-orthogonal and unstructured grids, the concept of transmissibility was maintained, probably for taking advantage of the simplicity in the programming. However, it is well known that for non-orthogonal grids, unstructured or not, the fluxes can not be exactly calculated using only two grid points, what precludes the use of the transmissibility for the flux calculation in such situations. On the other hand, it is common to find in the literature a recommendation that triangles, as used in unstructured grids should not have internal angles greater than $90^{\circ}$ in order to avoid the appearance of a negative transmissibility. It is shown that this is a misinterpretation of the transmissibility concept, since transmissibility is always a positive quantity.

Keywords: unstructured grids, transmissibility, petroleum reservoir simulation, element-based finite volume methods.
\end{abstract}

\section{Introduction}

The numerical techniques embodied in the pioneering industrial petroleum reservoir simulators employed finite-difference method with Cartesian grids. In this approach the connecting coefficients of a 5-point stencil in 2D can be written using the well know concept of transmissibility, $[1,2]$. The use of non-orthogonal curvilinear coordinates were also introduced seeking generality and flexibility of 
the numerical schemes, [3-5]. Defining each grid independently, with its coordinates given by the grid generator, as seen in Fig. 1, unstructured grids were introduced.

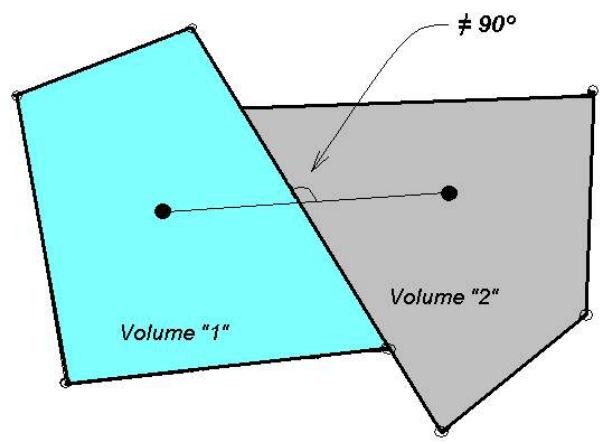

Figure 1: Non-orthogonal grid. Difficulties in evaluating the mass flux.

In this case, the line joining the two grid points is not orthogonal to the flux area. Therefore, if one insists in applying the transmissibility concept to this situation, errors will arise in the mass fluxes evaluation, since it is mathematically impossible to exactly calculate the mass flux using only two grid points. This error, of course, is a conceptual error, and does not disappear as the grid is refined. Despite this error, several traditional methods used in petroleum engineering, as reported by Sammon [6], employ two-point flux approximation schemes in order to reduce the computational cost and to simplify the code implementing. This paper addresses the two-grid point approximation issue and presents the basic ideas of a numerical scheme which perform exact fluxes calculation and preserves the conservation principles at control volume level. The method can mix triangular and quadrilateral elements. In this method, the transmissibility concept, according to its definition, is no longer valid. Therefore, the negative transmissibility, which is reported in the literature $[7,8]$ to appear in certain situations with triangular grids, is a misinterpretation of its concept. This is demonstrated by solving a well selected problem where this situation occurs.

\section{Transmissibility approach in reservoir simulation}

As mentioned, several reservoir simulation models use two-point flux approximation schemes. This is based on the physical idea that a flow of a quantity is directly proportional to a potential difference and inversely to the corresponding resistance to this flow. Therefore, the mass flux of a component between two adjacent grid-blocks $i$ and $j$ in the discrete solution of the transport equations is given, according to Heinemann and Brand [9], by

$$
Q_{i j}=\sum_{p=1}^{p}\left(\Lambda_{p} k\right)_{i j} \frac{A_{i j}}{h_{i j}}\left(\Phi_{j}-\Phi_{i}\right)_{p}
$$


where $\Lambda_{p}$ is the mobility of phase $p, \mathrm{P}$ is the number of phases; $k$ is the absolute permeability, $\Phi$ is the phase potential, $A_{i j}$ and $h_{i j}$ are, respectively, an flow area and a diffusion-like coefficient for the gradient determination at the interface. In Eq. (1), the terms which do not depend on of pressure and saturation can be grouped, resulting in

$$
Q_{i j}=T_{i j} \sum_{p=1}^{P}\left(\Lambda_{p}\right)_{i j}\left(\Phi_{j}-\Phi_{i}\right)_{p}
$$

where $T_{i j}$ is called transmissibility which is, therefore, defined as

$$
T_{i j}=k_{i j} \frac{A_{i j}}{h_{i j}}
$$

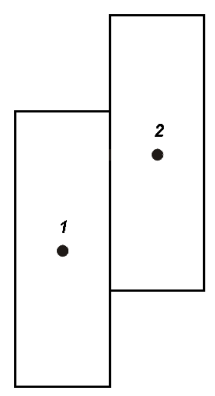

Figure 2: $\quad$ Transmissibility using the analogy with electrical conductance.

Eq. (3) requires the evaluation of the permeability (a physical property) at the control volume interfaces. As the domain may be heterogeneous, frequently with large differences in physical properties in adjacent grid-blocks, the definition of average properties at the interface can result in errors in the flux calculation. Therefore, the most appropriate procedure is to define the transmissibility for each grid block. For orthogonal grids with fully coincident interfaces this procedure leads to the exact flux determination, as already mentioned. On the other hand, for non-orthogonal grids, for grids with partial contact between the grid-blocks or with local refinement, the fluxes areas and lengths are not clearly defined. Even following the physical insight for this choice, the resulting flux will be non-exact. Applying electrical resistance concept, we obtain for a full contact area

$$
T_{12}=\frac{1}{\frac{1}{T_{1}}+\frac{1}{T_{2}}}=\frac{T_{1} T_{2}}{T_{1}+T_{2}}
$$

If there is partial contact between two grid-blocks, as shown in Fig. 2, the total transmissibility can be calculated as 


$$
T_{12}=\frac{A_{c}}{\frac{A_{1}}{T_{1}}+\frac{A_{2}}{T_{2}}}
$$

where $A_{c}$ is the contact surface, $A_{1}$ and $A_{2}$ are, respectively, the surface of each block, and $T_{1}$ and $T_{2}$ are their inner transmissibility. We can observe that in this case we chose to use the area $A_{c}$, the contact area, to calculate the transmissibility of grid-blocks 1 and 2, which is an approximation that will not lead to the exact value, even with grid refinement.

If one decides to use only two grid points, it must be clear that this procedure will always furnish a non-exact flux calculation. The choice of the flux area and the diffusion-like length may alleviate this problem by choosing physically consistent values for these parameters, Hegre et al. [10].

\section{The Element-based Finite Volume Method (EbFVM)}

\subsection{Fundamentals}

As reported by Tamin et al. [11], a great amount of research was dedicated in the last decade in evaluating the available tools for numerical reservoir simulation. In contrast, there were little efforts in developing new technologies and new approaches using conservative numerical schemes. In this section it is presented a numerical algorithm to be applied for simulating porous media flow with heterogeneities. It employs the ideas of Raw [12] applied to the Navier-Stokes equations. It belongs to the class of the Element-based Finite Volume Methods (EbFVM) with new features for mobility, relative and absolute permeability evaluation and local refinement near wells and/or faults. In this paper attention is devoted to clarify the alleged appearance of negative transmissibility when triangular grids with internal angles greater than $90^{\circ}$ are employed.
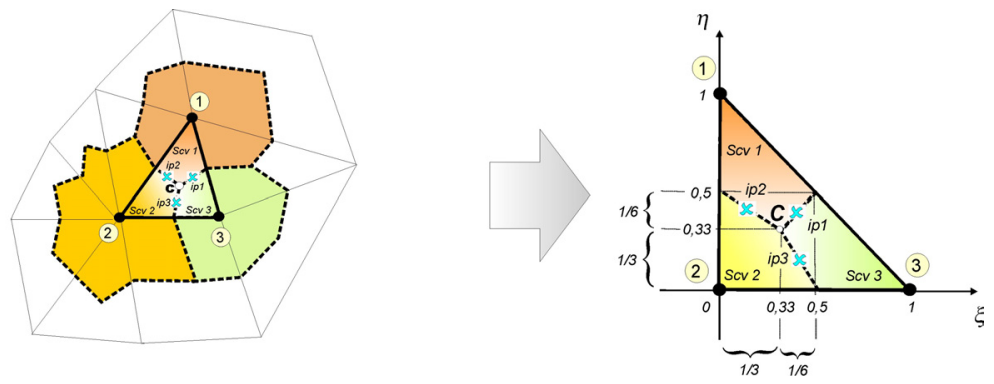

Figure 3: Elements and control volumes in triangular meshes.

In a finite volume methodology the domain is covered by non-overlapping control volumes where the balances are done, as shown in Fig. 3, where triangular elements and control volumes are identified. In the cell vertex construction, the control volumes are created joining the centre of the elements 
to its medians. The resulting control volume is formed by portions (sub-control volumes) of neighbouring elements. In this case, all fluxes at one specified integration point can be calculated using data from the element where the integration point lies. The saturation equation for a multiphase flow, not considering capillary pressure, is

$$
\frac{\partial}{\partial t}\left(\phi \frac{S_{m}}{B_{m}}\right)=\vec{\nabla} \cdot\left(\lambda_{m} \overline{\bar{k}} \vec{\nabla} p\right)+\tilde{q}_{m}
$$

where $m$ identifies the phase, $\mathrm{p}$ is the pressure common to the existing phases, $\lambda$ is the mobility of the phase, $\phi$ the porosity and $S$ the saturation of the phase. One is interested in evaluating the mass fluxes at the interfaces. The integration of divergent term in Eq. (6), yields

$$
\int_{S} \lambda_{m} \overline{\bar{k}} \vec{\nabla} p \cdot d \vec{S} \simeq \sum_{i p}\left(\lambda_{m} \overline{\bar{k}} \vec{\nabla} p \cdot \Delta \vec{S}\right)_{i p}
$$

corresponding to the evaluation at all integration points located at the surface of the control volume. The number of integration points depends on how many triangular elements contribute for forming the control volume where balances are performed. Integrating the remaining terms and collecting them, the approximate form of Eq. (6) appears as

$$
\left(\frac{S_{m}}{B_{m}}\right)_{P}=\left(\frac{S_{m}^{o}}{B_{m}^{o}}\right)_{P}+\left[\left(\bar{q}_{m}\right)_{P}+\sum_{i p}\left(\lambda_{m} \overline{\bar{k}} \vec{\nabla} p \cdot \Delta \vec{S}\right)_{i p}\right] \frac{\Delta t}{\phi_{P} \Delta V_{P}}
$$

in which the subscript $P$ refers to the control volume, while ip refers to the integration points located at the surface of the control volume.

The right-hand side term inside the bracket in Eq. (8) needs to be evaluated at the integration points, while pressure and saturation are available at the nodes. The mobility evaluation at the integration points is one of the key points in simulating multiphase flow in porous media, since it is the main responsible for the appearance of the well known grid orientation effects. Mobility is a function of the water saturation and when multiplied by the normal pressure gradient gives rise to the flow rate. This requires the specification of an adequate interpolation function which should take into account the direction of the flow. The traditional schemes perform a coordinate-oriented up-winding only, therefore, introducing the undesirable grid orientation effects. This matter is not in the scope of this work, and for now it suffices to mention that a type of flow direction up-winding interpolation is performed. Details about the method can be found in [13]. 
The evaluation of $(\vec{\nabla} p \cdot \Delta \vec{S})_{i p}$ is realized using the shape function defined for triangular elements. This is consistent since pressure is an elliptic term and its approximation using the bilinear shape functions

$$
\left\{\begin{array}{l}
N_{1}(\xi, \eta)=1-\xi-\eta \\
N_{2}(\xi, \eta)=\xi \\
N_{3}(\xi, \eta)=\eta
\end{array}\right.
$$

as

$$
p=\sum_{j=1}^{3} N_{j}(\xi, \eta) p_{j}
$$

keeps the consistency between the numerical approximation and physics. Therefore, the numerical approximation reads

$$
\sum_{i p}\left(\lambda_{m} \overline{\bar{k}} \vec{\nabla} p \cdot \Delta \vec{S}\right)_{i p}=\sum_{i p}\left(\lambda_{m} \overline{\bar{k}}\left(\frac{\partial p}{\partial x} \Delta y-\frac{\partial p}{\partial y} \Delta x\right)\right)_{i p}
$$

where the partial derivatives for pressure are obtained using the shape functions. Enforcing the global mass conservation and substituting all terms in Eq. (8) one obtains an equation for pressure determination. In this paper the IMPES methodology is used, whereby the pressure is solved implicitly and saturation explicitly.

\subsection{Mass flux calculation at the integration point in the EbFVM}

Eq. (11) allows the calculation of the mass flux at any integration point which belongs to a specified element. For example, the flow through integration point ip 2 in Fig. 3, is given by

$$
\left(\lambda_{m} \overline{\bar{k}} \vec{\nabla} p \cdot \Delta \vec{S}\right)_{i p 2}=\lambda_{i p 2}\left[T_{13}\left|\left(p_{3}-p_{1}\right)+T_{12}\right|\left(p_{2}-p_{1}\right)\right]
$$

demonstrating that the mass flux calculation requires one to take into account the pressure of the three grid points belonging to the element. This is obvious, of course, since the area where integration point ip2 lies is not orthogonal to the line joining grid points 1 and 2. Therefore, grid points 1, 2 and 3, must be considered. Inspecting Eq. (12), it becomes clear that it is not possible to define a transmissibility which will permit one to calculate the exact mass flux involving only two grid nodes. In Eq. (12) there are geometrical and physical information amalgamated in the coefficients $T_{13}$ and $T_{12}$, but they cannot be viewed as transmissibility according its definition. As already advanced, one is free to 
calculate the mass flux using only grid points 1 and 2 and defining $T_{12}$ as the transmissibility. It must be understood that this procedure, however, gives the wrong mass flux. It is possible to alleviate the errors by a better choice of the areas and lengths involved. Results for different choices and its influence on the error of the mass flux calculation can be found in [14]

\section{Negative transmissibility: does it exist?}

Fig. 4 depicts the domain for the reservoir simulation where three production wells with prescribed pressure at the left side of the domain, and two injection wells (oil and water) located in the top and bottom right, respectively, of the domain. In this problem the flow will be parallel to the $x$-axis. Water and oil viscosities are set identical and the flow is incompressible. The grid is constructed with 3 elements and 5 control volumes. Element 2, defined by nodes 1,2 and 3 is constructed such that it displays one internal angle more than $90^{\circ}$, what would cause the appearance of negative transmissibility. The appearance of a negative transmissibility would not be admissible, since transmissibility is always a positive quantity. This, as reported in the literature [7,8], would cause convergence problems. The recommendation, therefore, would be avoiding such elements when building the grid.

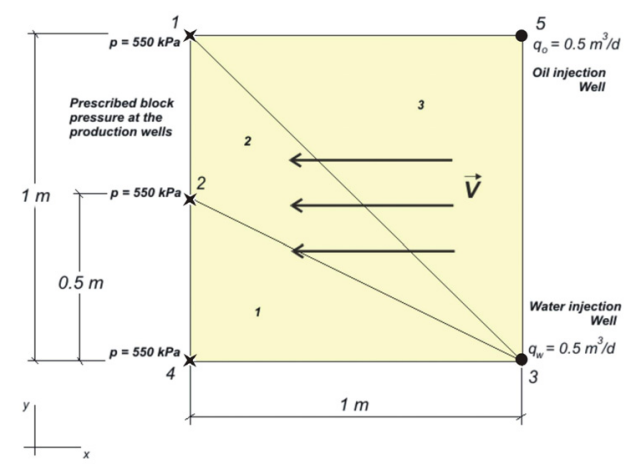

Figure 4: Idealized problem to show the negative transmissibility [14].

Element 2 and its fluxes areas connecting with sub-control volumes of neighbouring control volumes are presented in Fig. 5. Point B is chosen to lie in the same horizontal line of point $\mathrm{C}$. The mass flux through faces $\mathrm{AB}$ and $\mathrm{BC}$, entering the sub-control volume 1 is given by

$$
Q_{1}=Q_{A B}+Q_{B C}=\mathfrak{I}_{13_{S v c 1}}\left(p_{3}-p_{1}\right)+\mathfrak{\Im}_{12_{S v c 1}}\left(p_{2}-p_{1}\right)
$$

Since pressures at nodes 1 and 2 are equal, Eq. (13) results in

$$
Q_{1}=\mathfrak{I}_{13_{\text {Svcl }}}\left(p_{3}-p_{1}\right)
$$


The geometrical and physical coefficient in Eq. (14) is written as

$$
\mathfrak{I}_{13_{S v c 1}}=\lambda_{12} \tau_{A B}^{13}+\lambda_{13} \tau_{B C_{S v c 1}}^{13}
$$

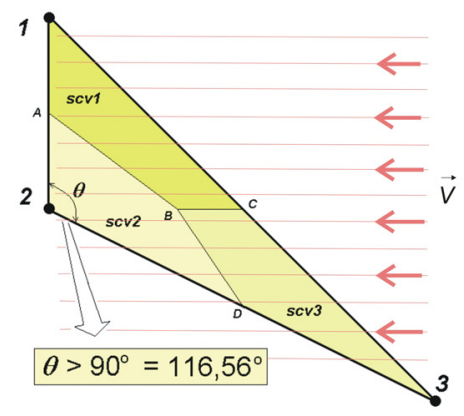

Figure 5: $\quad$ Element 2 with its fluxes areas identified.

The second term in the right hand side of Eq. (15) is zero, since the area normal of the flow of the segment $\mathrm{BC}$ is zero. Therefore, Eq. (15), reads

$$
Q_{1}=\lambda_{12} \tau_{A B}^{13}\left(p_{3}-p_{1}\right)
$$

Since the pressure difference is positive and the mass flux is negative, one obtains

$$
Q_{1}=\lambda_{12} \tau_{A B}^{13}\left(p_{3}-p_{1}\right)<0
$$

which demonstrates that

$$
\tau_{A B}^{13}<0
$$

This coefficient, as shown, can be negative and no longer can be interpreted as transmissibility, which is always a positive quantity. The reason for the existence of convergence problems, as reported in the literature, is linked with the fact that the equation for multiphase flows were obtained from the discretized equations for single phase flows, multiplying each term by the respective mobility. This, of course, ends up in a wrong discretized equation for multiphase flows, working properly only for constant viscosity and permeability. Details can be found in [14].

\section{Conclusions}

In this paper it was discussed important aspects related to the type of grids used in petroleum reservoir simulation. It became clear that only in presence of locally 
orthogonal grids the two-point approach yields exact results, precluding the use of transmissibility when non-orthogonal grids are employed, if exact fluxes are to be calculated.

It was also presented a flexible numerical scheme, the EbFVM (Element based Finite Volume Method), which can mix triangular and quadrilateral elements. The method, also referred as CVFEM in the literature, retains the geometric flexibility of the finite-element procedure and derives the governing discrete algebraic equations by using a conservation balance applied to discrete control volumes laid-out throughout the domain. The solution of a 2D problem demonstrates that the negative coefficient which appears when CVFEM is used with triangular grids with internal angle greater than $90^{\circ}$ is not transmissibility, as defined when two grid-blocks are used. This coefficient can be negative causing no difficulties for the convergence characteristics of the scheme.

\section{Acknowledgements}

The authors are grateful to ANP-Brazilian Agency for Petroleum and Energy and Petrobras for the partial support of this work.

\section{References}

[1] Todd, M. R., O'Dell, P. M. And Hirasaki, G. J., Methods for Increased Accuracy in Numerical Reservoir Simulators, SPE Journal, 515-530, 1972.

[2] Aziz, K. and Settari, A., Petroleum Reservoir Simulation, Applied Science Publishers, London, 1979.

[3] Heinemann, Z. E., Brand, C. W., Margit, M. and Chen, Y. M., Modeling Reservoir Geometry With Irregular Grids, SPE Reservoir Engineering, pp. 225-232, 1991.

[4] Rozon, B. J., A Generalized Finite Volume Discretization Method for Reservoir Simulation, SPE paper 18414 presented at the Reservoir Simulation Symposium, Houston, Texas, February 6-8, 1989.

[5] Maliska, C. R., Silva, A.F.C, Czesnat, A.O., Lucianetti, R.M., Maliska Jr., C.R., "Three-Dimensional Multiphase Flow Simulation in Petroleum Reservoirs using the Mass Fractions as Dependent Variables", Proceedings Fifth Latin American And Caribbean Petroleum Engineering Conference And Exhibition, Rio de Janeiro-RJ, Brasil, 1997.

[6] Sammon, P. H., Calculation of Convective and Dispersive Flows for Complex Corner Point Grids. Paper SPE 62929, Computer Modelling Group, Ltd., 2000.

[7] Fung, L. S., Hiebert, A. D. and Nghiem, L., Reservoir Simulation With a Control-Volume Finite-Element Method, SPE paper 21224 presented at the $11^{\text {th }}$ SPE Symposium on Reservoir Simulation, Anaheim, California, February 17-20, 1991.

[8] Sonier, F., Lehuen, P. and Nabil, R., Full-Field "Gas Storage Simulation Using a Control-Volume Finite Model”, SPE paper 26655 presented at 
the $68^{\text {th }}$ Annual Technical Conference and Exhibition, Houston, Texas, 36 October, 1993.

[9] Heinemann, Z. E. \& Brand, C. W., Gridding Techniques in Reservoir Simulation. $1^{\text {st }} / 2^{\text {nd }}$ Stanford Univ. \& Leoben Mining Univ. Reservoir Simulation Inf. Fórum, 1989.

[10] Hegre, T.M., Dalen, V., and Henriquez, A.," Generalized Transmissibilities for Distorted Grids in Reservoir Simulation", SPE 15622 .

[11] Tamin, M., Abou-kassem, J. H. and Farouq Ali, S. M., "Recent Developments in Numerical Simulation Techniques of Thermal Recovery", SPE paper 54096 presented at the SPE International Thermal Operations and Heavy Oil Symposium, Bakersfield, California, 17-19 March, 1999.

[12] Raw, M., "A New Control Volume Based Finite Element Procedure for the Numerical Solution of the Fluid Flow and Scalar Transport Equations, Ph.D. Thesis, University of Waterloo, Waterloo, Ontario, Canada, 1985.

[13] Maliska, C.R., "Computational Heat Transfer and Fluid Mechanics" , Editora Livros Técnicos e Científicos S/A, 464 pp. ,2004 (in portuguese).

[14] Cordazzo J., Simulação de Reservatórios de Petróleo utilizando o Método EbFVM e Solver Mutigrid, Ph.D. Thesis, Federal University of Santa Catarina, Mechanical Engineering Department, 2005 